\title{
Glitches in Southern Pulsars
}

Na Wang, R. N. Manchester ${ }^{2}$, R. Pace $^{2}$, M. Bailes ${ }^{3}$, V. M. Kaspi ${ }^{4}$, B. W. Stappers ${ }^{5}$, A. G. Lyne ${ }^{6}$

${ }^{1}$ Urumqi Astronomical Observatory, 40 South Beijing Road, Urumqi 830011, China

${ }^{2}$ Australia Telescope National Facility, CSIRO, PO Box 76, Epping NSW 1710, Australia

${ }^{3}$ Astrophysics and Supercomputing, Swinburne University of Technolgy, PO Box 218, Hawthorn Vic. 3122, Australia

${ }^{4}$ MIT, Center for Space Research 37-621, 70 Vassar St., Cambridge MA 0 2139, USA

${ }^{5}$ Mt Stromlo Observatory, ANU, Private Bag, Weston Creek ACT 2611, Australia

${ }^{6}$ University of Manchester, Jodrell Bank Observatory, Macclesfield, Cheshire SK11 9DL, UK

Abstract. Parkes timing observations of 31 mostly young pulsars over nearly nine years are described. A total of 29 glitches were detected, of which 19 are previously unreported. Twelve glitches were seen in PSR J1341-6220, making this the most frequently glitching pulsar known, and the largest known glitch was detected in PSR J1614-5047. Distributions of glitch parameters were investigated.

Observations were made using the Parkes 64-m radio telescope between 1990 January and 1998 December, mostly at frequencies around $1400 \mathrm{MHz}$. Both filterbank and correlator systems were used to provide the frequency resolution for dedispersion. TOAs resulting from this program were analysed using the most recent version of TEMPO (http://pulsar.princeton edu/tempo) which includes provision for fitting parameters to multiple glitches. A total of 31 pulsars were monitored during the program and 29 glitches were observed in ten pulsars.

We briefly describe here some of the more interesting results. A complete report on this work is given by Wang et al. (1999).

PSR J1048-5832: This pulsar suffered two large glitches of size $\sim 3 \times 10^{-6}$ and $\sim 0.7 \times 10^{-6}$ in 1993 February and 1997 December respectively. Observed frequency residuals are shown in Fig. 1. The expanded plot of $\Delta \nu$ (Panel b) clearly shows a third and much smaller glitch about $100 \mathrm{~d}$ before the large first glitch and also the quasi-exponential recovery from the larger glitches.

PSR J1341-6220: Twelve glitches were observed in this pulsar over 8.2 years, making it the most frequently glitching pulsar known. Four of the glitches have $\Delta \nu / \nu$ in the range $(0.7-1.7) \times 10^{-6}$, but the rest are small with $\Delta \nu / \nu \lesssim$ $0.1 \times 10^{-6}$. 
PSR J1048-5832

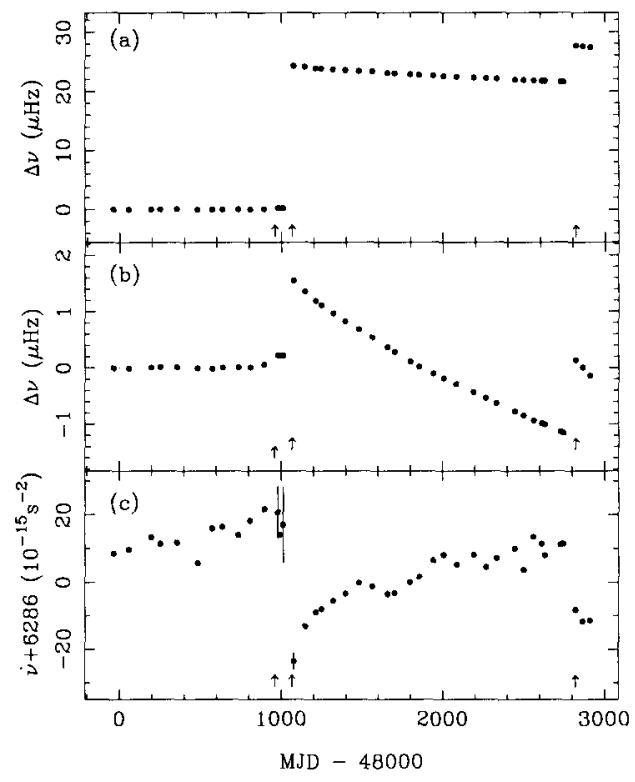

Figure 1. Glitches of PSR J1048-5832: variations of (a) frequency residual $\Delta \nu$ relative to the pre-glitch solution, (b) an expanded plot of $\Delta \nu$ where the mean increment after the second and third glitches have been removed, and (c) the variations of $\dot{\nu}$.

PSR J1614-5047: This young pulsar $\left(\tau_{c} \sim 7400 \mathrm{y}\right)$ suffered a massive glitch with $\Delta \nu / \nu \sim 6.5 \times 10^{-6}$ in 1995 June, the largest ever observed in any pulsar (cf. Shemar \& Lyne 1996). About half the glitch frequency increment decayed on a. long $(\sim 2000 \mathrm{~d})$ timescale. Evidence was found for a new class of irregularity in which the pulse frequency increases markedly over a few-day interval with an accompanying decrease in the magnitude of the frequency derivative.

Analysis of glitch parameters, both from this work and from previously published results, show that most glitches have a relative amplitude $\Delta \nu_{g} / \nu$ of between $10^{-8}$ and $10^{-6}$. Inter-glitch intervals range from $32 \mathrm{~d}$ to several thousand days and there is no correlation between the size of a glitch and the length of the preceding interval. In older pulsars, a smaller fraction of the frequency glitch decays, but there is little correlation between decay timescale and age.

\section{References}

Shemar, S. L. \& Lyne, A. G. 1996, MNRAS, 282, 677

Wang, N., Manchester, R. N., Pace, R., Bailes, M., Kaspi, V. M., Stappers, B. W. \& Lyne, A. G. 1999, MNRAS, submitted. 